\title{
Alterações farmacocinéticas de antirretrovirais em situações especiais: uma revisão narrativa
}

\author{
Antiretroviral pharmacokinetic changes in special situations: a narrative review \\ Cambios farmacocinéticos de los antirretrovirales en situaciones especiales: una revisión narrativa
}

Recebido: 03/09/2021 | Revisado: 10/09/2021 | Aceito: 13/09/2021 | Publicado: 14/09/2021

Gilcélia Correia Santos Bernardes

ORCID: https://orcid.org/0000-0002-2839-2443

Universidade Federal de São João Del-Rei, Brasil

E-mail: celiabiomedica@gmail.com

Thaís Lorenna Souza Sales

ORCID: https://orcid.org/0000-0002-1571-3850 Universidade Federal de São João Del-Rei, Brasil

E-mail: thaislorennass30@yahoo.com.br

Marco Túlio Alves Santos

ORCID: https://orcid.org/0000-0002-4334-0561

Universidade Federal de São João Del-Rei, Brasil

E-mail: marcotulioas@ hotmail.com

Bruna Gabriela Rocha

ORCID: https://orcid.org/0000-0003-3090-453X Universidade Federal de São João Del-Rei, Brasil E-mail: brunagabrielarocha@gmail.com

Anna Carolina de Moura Costa

ORCID: https://orcid.org/0000-0001-6813-9007 Universidade Federal de São João Del-Rei, Brasil E-mail: annacmourac@ hotmail.com

Adrielle Pieve de Castro

ORCID: https://orcid.org/0000-0002-1260-5619 Universidade Federal de São João Del-Rei, Brasil E-mail: adriellepieve@hotmail.com

Carlos Ananias Aparecido Resende

ORCID: https://orcid.org/0000-0003-1310-408X

Universidade Federal de São João Del-Rei, Brasil

E-mail: carlosresende.farmaceutico@gmail.com

Cristina Sanches

ORCID: https://orcid.org/0000-0002-8562-1337

Universidade Federal de São João Del-Rei, Brasil E-mail: csanches@ufsj.edu.br

\begin{abstract}
Resumo
O número de novos casos de infecção pelo HIV tem aumentado continuamente. No cenário mundial, nota-se a ampliação do acesso à terapia antirretroviral e, atualmente, 26 milhões de pessoas fazem uso de antirretrovirais. No Brasil, país que fornece acesso integral e gratuito aos antirretrovirais, cerca de 630 mil pessoas encontram-se em tratamento. O presente estudo tem o objetivo de descrever as alterações farmacocinéticas dos antirretrovirais em pacientes com condições fisiológicas distintas. Realizou-se um levantamento bibliográfico com estudos relativos ao uso de antirretrovirais em grupos específicos: extremos de idade (recém-nascidos e idosos), gravidez, doença hepática, doença renal crônica, doença cardiovascular, obesidade, alterações metabólicas, interações alimentares, uso e abuso de drogas lícitas e ilícitas e polimorfismo genético. Em determinadas situações, verificou-se alterações em parâmetros farmacocinéticos como absorção, distribuição, metabolismo e excreção, assim como, variações dos níveis plasmáticos dos antirretrovirais. Destaca-se nos grupos investigados, a importância do ajuste de dose para aumentar a efetividade dos antirretrovirais e proporcionar um tratamento mais adequado às pessoas vivendo com HIV.
\end{abstract}

Palavras-chave: Antirretrovirais; Farmacocinética; HIV.

\begin{abstract}
The number of new cases of HIV infection has been steadily increasing. In the world scenario, there is an increase in access to antiretroviral therapy and, currently, 26 million people use antiretrovirals. In Brazil, a country that provides full and free access to antiretrovirals, about 630 thousand people are undergoing treatment. This study aims to describe the pharmacokinetic changes of antiretrovirals in patients with different physiological conditions. A bibliographic survey was carried out with studies related to the use of antiretrovirals in specific groups: extremes of
\end{abstract}


age (newborns and elderly), pregnancy, liver diseases, chronic kidney disease, cardiovascular disease, obesity and metabolic disorders, dietary interactions, use and abuse of legal and illegal drugs and genetic polymorphism. In certain situations, changes in pharmacokinetic parameters such as absorption, distribution, metabolism and excretion were observed, as well as variations in plasma levels of antiretrovirals. In the investigated groups, the importance of dose adjustment is highlighted to increase the effectiveness of antiretrovirals, aiming to provide a more adequate treatment to people living with HIV.

Keywords: Antiretrovirals; Pharmacokinetic; HIV.

\section{Resumen}

El número de nuevos casos de infección por el VIH ha aumentado continuamente. En el escenario mundial, hay una expansión del acceso a la terapia antirretroviral y, en la actualidad, 26 millones de personas usan antirretrovirales. En Brasil, un país que brinda acceso pleno y gratuito a los medicamentos antirretrovirales, alrededor de 630 mil personas están en tratamiento. Este estudio tiene como objetivo describir los cambios farmacocinéticos de los fármacos antirretrovirales en pacientes con diferentes condiciones fisiológicas. Se realizó un relevamiento bibliográfico con estudios sobre el uso de antirretrovirales en grupos específicos: extremos de edad (recién nacidos y ancianos), embarazo, enfermedad hepática, enfermedad renal crónica, enfermedad cardiovascular, obesidad, alteraciones metabólicas, interacciones alimentarias, uso y abuso de Drogas legales e ilegales y polimorfismo genético. En determinadas situaciones, se produjeron cambios en parámetros farmacocinéticos como absorción, distribución, metabolismo y excreción, así como variaciones en los niveles plasmáticos de antirretrovirales. En los grupos investigados, se destaca la importancia del ajuste de dosis para aumentar la eficacia de los antirretrovirales y proporcionar un tratamiento más apropiado para las personas que viven con el VIH.

Palabras clave: Antirretrovirales; Farmacocinética; VIH.

\section{Introdução}

As infecções causadas por retrovírus são de grande importância clínica e apresentam uma elevada prevalência. No mundo, a infecção pelo vírus da imunodeficiência humana (HIV) atinge 38 milhões de pessoas, sendo que o número de novas infecções chegou a 1,7 milhões em 2019. Neste mesmo ano, foram registradas 690 mil mortes relacionadas à síndrome da imunodeficiência adquirida (AIDS), comparadas a 1,1 milhões em 2010. O acesso à terapia antirretroviral teve um aumento significativo, passando de 7,7 milhões em 2010 para 26 milhões em 2020 (UNAIDS, 2020). No Brasil, o acesso integral e gratuito aos antirretrovirais foi instituído na década de 1990 e, atualmente, cerca de 630 mil pessoas encontram-se em tratamento (Brasil, 1996; UNAIDS, 2019a). A terapia antirretroviral vem interferindo positivamente na expectativa de vida dos pacientes (Brito, 2012). No entanto, a efetividade dos antirretrovirais, muitas vezes, pode ser comprometida devido à ausência de individualização terapêutica para grupos com condições fisiológicas específicas.

Pacientes em terapia antirretroviral que apresentam condições fisiológicas distintas e comorbidades específicas, devem ser avaliados quanto à necessidade do ajuste de dose. Fatores como extremos de idade, gravidez, doença hepática, doença renal crônica, doença cardiovascular, obesidade, alterações metabólicas, interações alimentares, uso e abuso de drogas lícitas e ilícitas e polimorfismo genético são capazes de modificar a farmacocinética dos antirretrovirais. Portanto, o ajuste de dose poderá ser requerido para evitar concentrações subterapêuticas responsáveis pela falha na supressão viral, assim como, concentrações supraterapêuticas capazes de resultar no aumento da toxicidade (Spina, Santoro, \& D’Arrigo, 2008).

No que se refere à farmacocinética, características referentes à solubilidade dos fármacos são de suma importância (Winter, 2004), sendo classificados de acordo com sua natureza hidrofílica ou lipofílica (Quadro 1). Os fármacos hidrofílicos são facilmente solubilizados nos fluidos corporais, no entanto, a absorção torna-se limitada devido à lipofílicidade das membranas biológicas. Já os fármacos lipofílicos apresentam maior capacidade de difusão e menor solubilidade nos fluidos corporais. Logo, para obter um maior potencial de absorção e alcançar a circulação sistêmica com mais facilidade, é fundamental que os fármacos apresentem um balanço adequado entre seu caráter lipofílico e demais propriedades que garantam sua solubilidade. Com relação à distribuição, sabe-se que os fármacos lipofílicos apresentam grande capacidade de atravessar barreiras, sendo distribuídos em maior extensão pelo organismo, enquanto, os fármacos hidrofílicos apresentam uma distribuição limitada. Nos processos de metabolismo e excreção, os fármacos lipofílicos passam pela etapa de 
biotransformação em que são convertidos em moléculas mais hidrofílicas, facilitando assim, sua eliminação. Neste aspecto, os fármacos hidrofílicos contam com uma rápida eliminação, quando se trata da via de excreção renal (Thapa, Choi, Kim, \& Yong, 2017; Yellepeddi, 2016). Vale ressaltar que os antirretrovirais apresentam características hidrossolúveis em sua maioria (NCBI, 2019).

Ao considerar a relevância dos estudos farmacocinéticos e suas contribuições para a aplicação da individualização terapêutica na prática clínica, essa revisão tem por objetivo demonstrar as principais alterações farmacocinéticas dos antirretrovirais em pacientes com condições fisiológicas distintas. Foram abordados extremos de idade (recém-nascidos e idosos), gravidez, doença hepática, doença renal crônica, doença cardiovascular, obesidade, alterações metabólicas e polimorfismo genético.

Quadro 1. Mecanismo de ação e solubilidade dos antirretrovirais.

\begin{tabular}{|c|c|c|}
\hline Mecanismo de Ação & Hidrossolúveis & Lipossolúveis \\
\hline $\begin{array}{l}\text { Inibidor da Transcriptase } \\
\text { Análogo de Nucleosídeo }\end{array}$ & $\begin{array}{l}\text { - Abacavir } \\
\text { - Estavudina } \\
\text { - Lamivudina } \\
\text { - Tenofovir } \\
\text { - Zidovudina }\end{array}$ & • Didanosina \\
\hline $\begin{array}{l}\text { Inibidor da Transcriptase Reversa Não } \\
\text { Análogo de Nucleosídeo }\end{array}$ & $\begin{array}{l}\cdot \text { Efavirenz } \\
\text { - Etravirina } \\
\text { - Nevirapina }\end{array}$ & $\cdot \mathrm{N} / \mathrm{A}$ \\
\hline Inibidor da Protease & $\begin{array}{l}\text { - Amprenavir } \\
\text { - Atazanavir } \\
\text { - Darunavir } \\
\text { - Fosamprenavir } \\
\text { - Indinavir } \\
\text { - Nelfinavir }\end{array}$ & $\begin{array}{l}\text { - Lopinavir } \\
\text { - Ritonavir } \\
\text { - Saquinavir } \\
\text { - Tipranavir }\end{array}$ \\
\hline Inibidor da Proteína de Fusão & $\cdot \mathrm{N} / \mathrm{A}$ & • Enfuvirtida \\
\hline Inibidor da Integrase & $\begin{array}{l}\text { - Dolutegravir } \\
\text { - Raltegravir }\end{array}$ & $\cdot \mathrm{N} / \mathrm{A}$ \\
\hline Inibidor de Entrada & - Maraviroc & $\cdot \mathrm{N} / \mathrm{A}$ \\
\hline
\end{tabular}

Fonte: National Center for Biotechnology Information - NCBI, 2019. N/A: não se aplica.

\section{Metodologia}

A revisão de literatura narrativa da qual se trata este estudo, apresenta uma análise ampla da literatura sobre as alterações farmacocinéticas de antirretrovirais em situações especiais. A revisão abrangeu artigos científicos disponíveis nas bases de dados: PubMed, Scielo (Scientific Eletronic Library Online), Scopus, Web of Science e Google acadêmico. Também foram utilizados relatórios do ministério da saúde e da UNAIDS.

\section{Resultados e Discussão}

O presente estudo demonstra as alterações farmacocinéticas de antirretrovirais em diferentes ocasiões, sendo apresentadas em forma de tópicos. A Tabela 1 apresenta de forma sintetizada as principais alterações farmacocinéticas em situações especiais e o impacto causado em diferentes classes de antirretrovirais. 
Tabela 1. Alterações farmacocinéticas dos antirretrovirais em situações especiais.

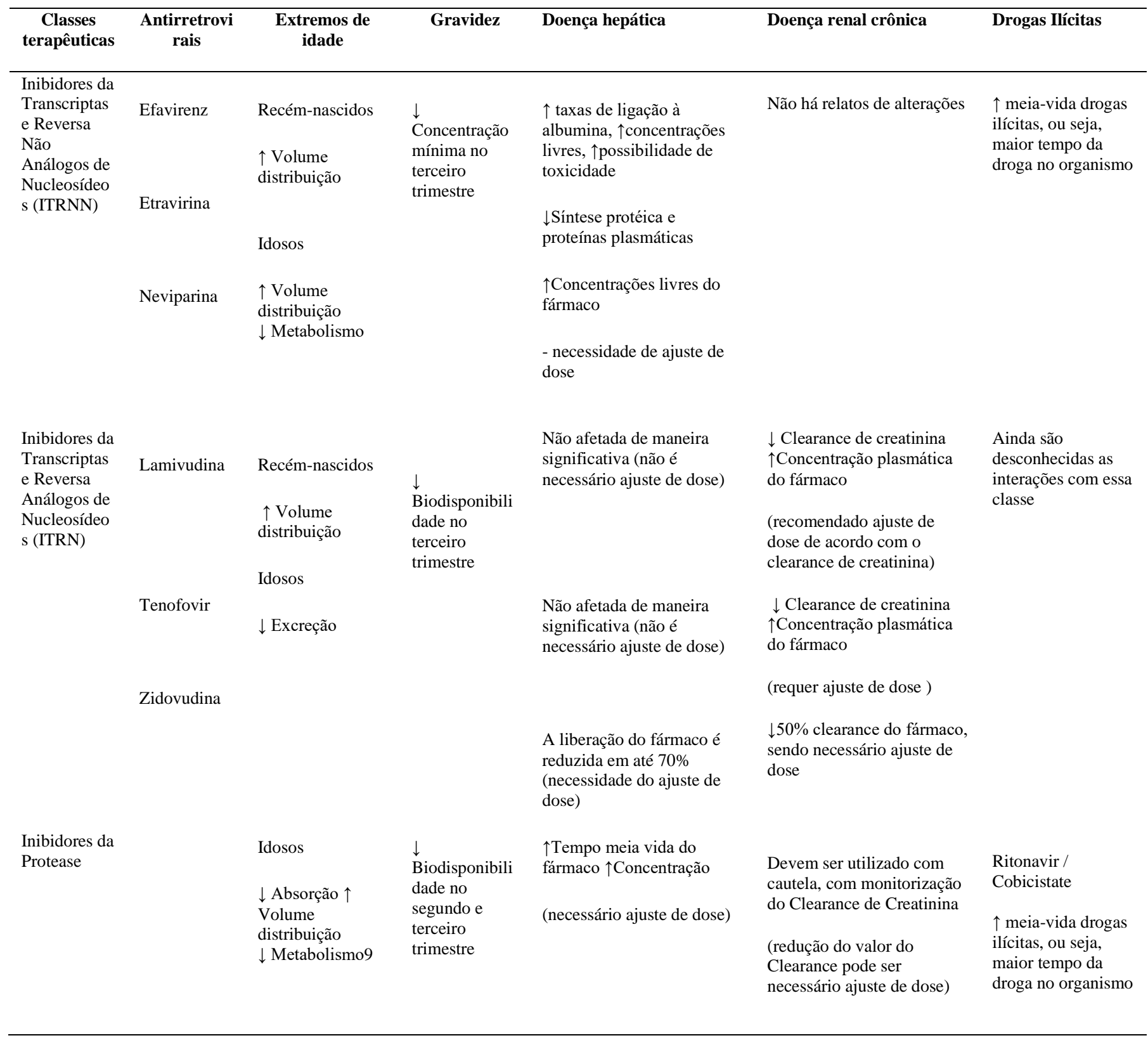

Fonte: Gilbert et al., (2015); Nuh, (2020); Pacifici, (2005); Schoen et al., (2013); Sulkowski et al., (2002).

\section{Extremos de idade}

A exposição ao HIV em recém-nascidos pode ocorrer em 3 fases distintas, ainda no útero, durante o parto ou através da amamentação (AIDSINFO, 2019). A terapia antirretroviral é indicada para recém-nascidos expostos ao HIV devido ao elevado risco de morbidade e mortalidade (Canals, Masiá, \& Gutiérrez, 2018). O início precoce da terapia nas primeiras horas de vida pode melhorar significativamente o resultado do tratamento (Canals et al., 2018; Kim, Ahmed, \& Abrams, 2016). Um estudo realizado em crianças infectadas pelo HIV revela que a terapia antirretroviral precoce foi capaz de reduzir a mortalidade em 76,0\% e a progressão do HIV em 75,0\% (Violari et al., 2008). Em paralelo, nota-se que o número de idosos com HIV tem aumentado rapidamente e, estima-se que em 2016, aproximadamente metade das pessoas vivendo com HIV nos Estados Unidos e áreas dependentes, tinham mais de 50 anos de idade (CDC, 2017). No Brasil, o aumento nos casos de HIV em pessoas com idade acima de 50 anos foi de aproximadamente 108,0\%, entre os anos de 2010 e 2019 (BRASIL, 2019). 
Indivíduos nos extremos de idade apresentam características peculiares nos aspectos fisiológicos que podem afetar os parâmetros farmacocinéticos dos medicamentos (Benedetti, Whomsley, \& Canning, 2007). Os recém-nascidos estão em constantes mudanças fisiológicas devido ao rápido desenvolvimento e processo de maturação dos sistemas orgânicos (Ku \& Smith, 2011), enquanto os idosos apresentam o declínio de funções fisiológicas inerentes ao envelhecimento (Stader et al., 2019), sendo ambas as condições capazes de comprometer a farmacocinética dos antirretrovirais (Courlet et al., 2019; Pacifici, 2005).

\section{Recém-nascidos}

Os dados sobre terapia antirretroviral em recém-nascidos são escassos. As mudanças fisiológicas que acontecem com elevada frequência em neonatos, principalmente na função renal, tornam a definição da dosagem ideal mais complexa (Rakhmanina \& Phelps, 2012).

Em recém-nascidos pré-termos, o único fármaco antirretroviral com recomendações de dosagem é a zidovudina (AIDSINFO, 2019; Canals et al., 2018), sendo sugerido o ajuste de dose devido à baixa depuração (dose inicial reduzida, com aumento de dose, após 2 semanas de vida) (Capparelli et al., 2003). Nos recém-nascidos a termos ocorre uma elevação das enzimas responsáveis pela glucuronidação da zidovudina nas primeiras semanas de vida e, com isso, é necessário ajuste de dose do fármaco após 6 semanas de vida (Canals et al., 2018). Com relação à lamivudina, a biodisponibilidade é descrita como menor quando se compara recém-nascidos com bebês mais velhos. Similarmente, o abacavir também possui baixa biodisponibilidade e depuração ao comparar recém-nascidos com crianças maiores (King, Kimberlin, Aldrovandi, \& Acosta, 2002). No caso da estavudina, verifica-se que os níveis de exposição em recém-nascidos são compatíveis aos observados em crianças maiores. Já a exposição à didanosina em recém-nascidos mostra-se superior quando comparados às crianças maiores (Rongkavilit et al., 2001).

\section{Idosos}

O envelhecimento resulta em alterações fisiológicas que podem afetar a farmacocinética dos antirretrovirais (Schoen, Erlandson, \& Anderson, 2013). Os idosos apresentam alterações na composição corporal, função hepática e renal que podem por consequência afetar o volume de distribuição, o metabolismo e a excreção dos fármacos (Mangoni \& Jackson, 2004). Alterações como elevação do pH gástrico, retardo no esvaziamento gástrico e diminuição do fluxo sanguíneo esplâncnico diminuem a taxa de absorção dos inibidores de protease, inibidores da transcriptase reversa não análogos de nucleosídeos e inibidores de fusão. $\mathrm{O}$ volume de distribuição dos antirretrovirais pertencentes a essas classes terapêuticas também é alterado em função de características muito comuns em idosos, como redução da albumina, aumento da gordura corporal, redução da água e queda da atividade de proteínas de transporte. Além disso, o declínio da função hepática e renal são capazes de alterar o metabolismo e a excreção desses fármacos (Guaraldi, Pintassilgo, Milic, \& Mussini, 2018). Estudos envolvendo pessoas idosas vivendo com HIV, demonstram ainda uma elevação da biodisponibilidade de antirretrovirais como tenofovir, lamivudina e emtricitabina (De Sousa Mendes \& Chetty, 2019), maior exposição ao atazanavir e elevação da concentração mínima de lopinavir (Jourjy, Dahl, \& Huesgen, 2015).

\section{Gravidez}

A ampliação da terapia antirretroviral em gestantes tem contribuído para evitar a transmissão vertical. Neste cenário, notou-se uma redução de aproximadamente 41,0\% na incidência de infecções pelo HIV em crianças entre os anos de 2010 (280 mil crianças infectadas) e 2018 (160 mil crianças infectadas) (UNAIDS, 2019b). 
Durante a gestação, ocorrem alterações fisiológicas que resultam em mudanças nos parâmetros farmacocinéticos (Quadro 2). Ainda são escassos os estudos destinados a avaliar o impacto de tais alterações durante o uso de antirretrovirais, assim como, o ajuste de dose necessário no período gestacional (Dawes Bsc \& Chowienczyk Bsc, 2001).

Quadro 2 Alterações fisiológicas na gravidez e seu impacto na farmacocinética.

\begin{tabular}{|l|l|}
\hline \multicolumn{1}{|c|}{ Parâmetros farmacocinéticos } & \multicolumn{1}{c|}{ Alterações fisiológicas } \\
\hline Absorção & $\begin{array}{l}\text { Enjoos frequentes resultam na redução da } \\
\text { absorção. } \\
\text { Elevação do pH gástrico ocasiona a redução da } \\
\text { absorção. }\end{array}$ \\
\hline Distribuição & $\begin{array}{l}\text { Aumento do volume de água corporal eleva o } \\
\text { volume distribuição de fármacos hidrofílicos. } \\
\text { Aumento da gordura corporal causa elevação do } \\
\text { volume de distribuição de fármacos hidrofóbicos. }\end{array}$ \\
\hline Metabolismo & $\begin{array}{l}\text { Alterado devido à elevação dos hormônios } \\
\text { sexuais. }\end{array}$ \\
\hline Excreção & $\begin{array}{l}\text { Elevação da depuração renal, aumenta a } \\
\text { eliminação de fármacos excretados pelos rins. }\end{array}$ \\
\hline
\end{tabular}

Fonte: Costantine (2014); Pariente et al. (2016).

As alterações farmacocinéticas podem prejudicar a efetividade de diversos medicamentos, inclusive dos antirretrovirais e, portanto, doses mais altas podem ser necessárias para se alcançar o efeito desejado (Gilbert, Darin, Scarsi, \& McLaughlin, 2015). Estudos demonstram que as alterações fisiológicas presentes na gestação não afetam de forma significativa a farmacocinética de alguns inibidores da transcriptase reversa análogos de nucleosídeos, como o abacavir e a zidovudina, não sendo recomendado o ajuste de dose para estes fármacos (Best et al., 2006; Connor et al., 1994). No entanto, verifica-se que a lamivudina e o tenofovir, também pertencentes à essa classe, apresentam maior depuração durante a gestação, indicando assim, a importância da individualização terapêutica (Benaboud, Hirt, et al., 2012; Benaboud, Tréluyer, et al., 2012). Similarmente, antirretrovirais como dolutegravir, maraviroque e efavirenz apresentam redução da biodisponibilidade em gestantes, especialmente no terceiro trimestre da gestação, o que implica na necessidade do ajuste de dose (Aidsinfo, 2018).

\section{Doença hepática}

As doenças hepáticas representam cerca de $13,0 \%$ a $18,0 \%$ de todas as causas de morte nas pessoas vivendo com HIV (Kaspar \& Sterling, 2017). O HIV induz lesões hepáticas por meio de interações diretas com células hepáticas, as quais se cruzam com células não infectadas e resultam na disseminação das lesões por todo o fígado. Os efeitos pró-apoptóticos do HIV nos hepatócitos destacam-se como os principais responsáveis pelo desenvolvimento dos processos de inflamação e fibrose no fígado (Ganesan, Poluektova, Kharbanda, \& Osna, 2018). Além disso, é comum a presença de coinfecção com os vírus da hepatite B e C, visto que compartilham as mesmas vias de transmissão (Kaspar \& Sterling, 2017). Outro fator de grande relevância consiste na toxicidade ocasionada pela terapia antirretroviral. Estudos demonstram que até 10,0\% das pessoas em uso de antirretrovirais apresentam hepatotoxicidade grave (Terada \& Hira, 2015), sendo que os mecanismos descritos envolvem reações de hipersensibilidade, toxicidade direta do fármaco e/ou seu metabólito, toxicidade mitocondrial e síndrome inflamatória da reconstituição imune (SIR) (Verma \& Kaplowitz, 2009). Os inibidores da transcriptase reversa análogos de nucleosídeos (ITRN) são associados à toxicidade mitocondrial, em função de sua capacidade de inibir a DNA polimerase 
gama. Já os inibidores da transcriptase reversa não análogos de nucleosídeos (ITRNN) podem causar hepatotoxicidade por reação de hipersensibilidade ou por toxicidade direta (Terada \& Hira, 2015).

A infecção pelo HIV, assim como, o potencial hepatotóxico de determinados antirretrovirais, são capazes de ocasionar níveis anormais de enzimas hepáticas (Núñez, 2010; Ritschel \& Kearns, 2009). A hepatite crônica está entre os danos mais comuns causados pelos antirretrovirais, podendo estar associada à presença de anomalias metabólicas ou à toxicidade direta (Araújo \& Ehrhardt, 2015). Neste caso, a combinação terapêutica utilizando inibidores da transcriptase reversa análogos de nucleosídeos e inibidores de protease tem sido considerada como uma opção de tratamento capaz de minimizar os danos hepáticos (Ishizaki et al., 2009; Pezzani, Resnati, Di Cristo, Riva, \& Gervasoni, 2016; J. de Souza \& Storpirtis, 2004).

O grau de dano hepático determinará o nível de alterações na síntese e nos sítios de ligação das proteínas plasmáticas. Em casos críticos, fatores como decréscimo da albumina sérica, aparecimento de proteínas plasmáticas defeituosas e acúmulo de compostos endógenos como a bilirrubina, são capazes de alterar a taxa de ligação dos fármacos às proteínas plasmáticas e resultar na redução do volume de distribuição dos antirretrovirais. Fármacos como o efavirenz, que possuem altas taxas de ligação à albumina, apresentam maiores concentrações livres, aumentando assim, a possibilidade de toxicidade (Sulkowski, Thomas, Mehta, Chaisson, \& Moore, 2002). Nos casos em que a circulação esplênica é afetada (ascite), o volume de distribuição de fármacos hidrossolúveis, como a zidovudina e a lamivudina, é aumentado e pode ocorrer ainda redução do fluxo hepático, afetando diretamente a biodisponibilidade de fármacos com extensivo efeito de primeira passagem (Medeiros \& Ferreira, 1998).

Adicionalmente, pessoas infectadas pelo HIV fazem uso de outros fármacos com potencial de toxicidade hepática, como por exemplo, antimicrobianos (Araújo \& Ehrhardt, 2015; Ritschel \& Kearns, 2009). O grande problema é que a longo prazo, a hepatotoxicidade presente em vários esquemas terapêuticos pode resultar em danos irreversíveis ao paciente (Pezzani et al., 2016). Por isso, faz-se necessário conhecer as alterações fisiopatológicas em pacientes com doença hepática e suas consequências sobre a farmacocinética dos antirretrovirais, assim como, o potencial de toxicidade da terapia antirretroviral nesse grupo específico, a fim de que sejam realizados os ajustes de dose necessários em cada situação para garantia de efetividade e segurança no uso desses fármacos.

\section{Doença renal crônica}

Evidências indicam um elevado risco de doença renal crônica em pessoas vivendo com HIV. Atualmente, a prevalência global de doença renal crônica nessa população é de 4,8\% a 12,3\% (Ekrikpo et al., 2018). Acredita-se que o desenvolvimento de doença renal crônica está associado à própria infecção, coinfecções, comorbidades, predisposição genética e como consequência da exposição à terapia antirretroviral a longo prazo.

Em virtude do alto potencial dos antirretrovirais em ocasionar ou agravar lesões renais, torna-se imprescindível que a função renal e os fatores de risco para doença renal crônica sejam avaliados antes do início do tratamento (Swanepoel et al., 2018). A exposição cumulativa aos antirretrovirais como tenofovir, indinavir, atazanavir, lopinavir, ritonavir e, menos evidentemente, efavirenz, abacavir, zidovudina ou estavudina, está associada ao aumento do risco de doença renal crônica (Mocroft et al., 2010). O tenofovir é o principal antirretroviral descrito nos casos de lesão tubular renal (Ellis, 2017; Nunes, 2016). Há evidências de que a toxicidade desse fármaco pode levar até mesmo ao desenvolvimento da Síndrome de Fanconi (Hall, Hendry, Nitsch, \& Connolly, 2011). Estudos realizados no Brasil, demostram que mudanças na taxa de filtração glomerular relacionadas ao uso de tenofovir, ainda que pequenas, tendem a persistir e se intensificar com o tempo e, além disso, nota-se uma maior prevalência de doença renal crônica em pacientes tratados com tenofovir em relação aos não tratados com outros fármacos (Menezes et al., 2011; Neto et al., 2016). Nos casos em que há confirmação de doença renal crônica, recomenda-se a descontinuação do tratamento com o tenofovir (Brasil, 2010). Adicionalmente, sabe-se que associações entre 
os antirretrovirais podem contribuir para o desenvolvimento de nefropatias (Friedl, Aprile, Fino, Barbosa, \& Belasco, 2009) e, neste caso, a combinação de tenofovir com inibidores de proteases (IP) é capaz de potencializar esse efeito tóxico. Tal situação é atribuída ao aumento da concentração tubular do fármaco por competição pelos sítios de excreção, uma vez que a administração conjunta desses antirretrovirais relaciona-se à redução do clearence de creatinina quando comparada à administração de esquemas contendo inibidores de transcriptase reversa não análogos de nucleosídeos (Nunes, 2016; Post \& Holt, 2009).

Os rins desempenham um papel importante na excreção dos fármacos e, portanto, sempre que detectada a presença de alterações na função renal, é de fundamental importância o ajuste de dose dos antirretrovirais excretados por via renal, através da estimativa do clearance de creatinina (redução da dose diária, alteração do regime de dose) (Reynes, Pevriere, De Boever, \& Le Moing, 2003). A maioria dos inibidores da transcriptase reversa análogos de nucleosídeos são excretados inalterados pelos rins e, portanto, o ajuste das doses é necessário para pacientes com taxa de filtração glomerular inferior a $60 \mathrm{~mL} / \mathrm{min}$. Os antirretrovirais pertencentes à essa classe terapêutica são fortemente ligados às proteínas plasmáticas, apresentam peso molecular baixo a médio e podem ser facilmente removidos por diálise. Assim, torna-se necessária suplementação da dose após a diálise, a fim de evitar concentrações subterapêuticas. Neste aspecto, vale ressaltar que antirretrovirais como os inibidores de protease, com exceção do indinavir, os inibidores da transcriptase reversa não análogos de nucleosídeos, com exceção da nevirapina e os inibidores de fusão são eliminados principalmente pelo fígado e, portanto, não requerem ajuste de dose (Naicker, Rahmanian, \& Kopp, 2015).

Adicionalmente, sabe-se que o desenvolvimento de doença renal crônica resulta em múltiplas alterações fisiopatológicas, como por exemplo, redução do esvaziamento gástrico (30,0\% pacientes com DRC), aumento do pH gástrico, alterações da composição corporal (edema), o que pode comprometer outros parâmetros farmacocinéticos, além da excreção (Schijvens, Wildt, \& Schreuder, 2020). Desta forma, observa-se a importância de se considerar as diversas alterações que ocorrem na farmacocinética dos antirretrovirais em pacientes com doença renal crônica, com o intuito de otimizar a farmacoterapia nessa importante condição clínica.

\section{Doença cardiovascular}

Em pessoas vivendo com HIV, doenças cardiovasculares, como cardiomiopatia, endocardite, miocardite, pericardite e insuficiência cardíaca podem ser impulsionadas por processos inflamatórios e disfunções imunológicas provenientes da replicação viral. Fatores de risco como idade avançada, histórico familiar, sedentarismo, dislipidemia, obesidade, hipertensão, diabetes, resistência à insulina e tabagismo também são responsáveis pelo desenvolvimento de doenças cardiovasculares nessa população específica (Chastain, Henderson, \& Stover, 2015; Sudano et al., 2006; Triant, 2013).

Além disso, graves distúrbios metabólicos resultantes da terapia antirretroviral, têm sido associados a um maior risco de doença arterial coronariana e infarto agudo do miocárdio (Chastain et al., 2015). De acordo com Hürlimann e colaboradores (2006), os inibidores de protease como atazanavir, lopinavir e ritonavir têm sido associados a alterações metabólicas importantes como resistência à insulina, anormalidades no metabolismo lipídico, lipodistrofia e consequente aumento da calcificação da artéria coronária. Nesse contexto, para os pacientes com risco cardiovascular superior à $20 \%$ pelo Escore de Framingham, recomenda-se a prescrição de terapia com tenofovir em preferência à zidovudina e terapia sem inibidores de protease com o intuito de minimizar os danos cardiovasculares (Raposeiras-Roubín \& Triant, 2016).

As alterações fisiopatológicas mais comumente observadas nas doenças cardiovasculares podem modificar a cinética de inúmeros fármacos, afetando todas as fases do processo farmacocinético. Geralmente, pacientes com doenças cardíacas apresentam redução do fluxo sanguíneo esplênico, edema intestinal, redução do esvaziamento gástrico e motilidade intestinal, ocasionando no atraso e redução da absorção dos fármacos. Na insuficiência cardíaca, a redução no débito cardíaco pode levar 
à redução do fluxo sanguíneo para os tecidos e, consequente, alteração no volume de distribuição. O edema também consiste em uma condição capaz de alterar o volume de distribuição, sendo possível observar o aumento na distribuição dos fármacos hidrossolúveis. A hipoproteinemia reduz a ligação dos fármacos às proteínas plasmáticas, aumentando a fração livre disponível e o volume de distribuição dos fármacos com alta afinidade às proteínas plasmáticas. O aumento da a1-glicoproteína ácida, frequente em condições como a fase aguda da necrose miocárdica, pode resultar no aumento das concentrações plasmáticas totais dos fármacos altamente ligados à essa proteína. Adicionalmente, a redução do fluxo sanguíneo hepático e renal em pacientes com insuficiência cardíaca provocam a redução do metabolismo hepático e excreção renal dos fármacos, respectivamente (Rodighiero, 1989). Em geral, a seleção da dose dos antirretrovirais para os pacientes com disfunções cardíacas deve ser cautelosa, tendo em vista as inúmeras alterações fisiopatológicas e suas consequências sobre a farmacocinética.

\section{Obesidade e alterações metabólicas}

Considerada como um risco para diversas doenças e complicações de saúde, a obesidade pode afetar a farmacocinética de inúmeros fármacos (Jain et al., 2011). Pessoas obesas apresentam maior variação na composição corporal e circulação sanguínea ao longo do corpo quando comparadas com pessoas não obesas (Smit, Hoogd, Brüggemann, \& Knibbe, 2018). Dessa maneira, a obesidade e demais alterações metabólicas devem ser preocupações para os mais diversos tipos de tratamentos, inclusive com antirretrovirais. Embora a população de indivíduos obesos esteja crescendo em todo o mundo, ainda há pouca orientação sobre como realizar o ajuste de dose quando esses indivíduos necessitam de intervenção farmacológica (Madelain et al., 2017).

Classes de antirretrovirais, como os inibidores não nucleosídeos da transcriptase reversa, os inibidores de protease e os inibidores da integrase apresentam fármacos com características lipofílicas que são susceptíveis à difusão e aprisionamento ao tecido adiposo, tendo menor concentração plasmática e consequentemente atividade antiviral reduzida (Madelain et al., 2017). 2017). Mesmo a molécula do tenofovir sendo hidrofílica, seu pró-fármaco ester, tenofovir disoproxil, é lipofílico. O efavirenz também é um fármaco lipofílico e apresenta alta ligação à albumina (99,5\%) (Madelain et al., 2017).

Uma elevada afinidade pelo tecido adiposo foi demonstrada, com concentrações até 100 vezes maiores do que no plasma (Dupin et al., 2002). Consequências da obesidade no organismo, podem interferir na concentração plasmática de antirretrovirais como o lopinavir, que apesar se ligar amplamente às proteínas plasmáticas, sofre metabolismo rápido pelas isoenzimas do citocromo $\mathrm{P} 450$, que possuem sua atividade reduzida devido à mudança na função hepática causada pela obesidade (Madelain et al., 2017), reduzindo assim a sua metabolização. O raltegravir é uma molécula lipofílica, mas apresenta reduzida solubilidade em solução aquosa ácida, tendo sua absorção gastrointestinal dependente em grande parte do $\mathrm{pH}$ gástrico. Cerca de 50,0\% dos pacientes obesos, sofrem com a doença do refluxo gastroesofágico (Chang \& Friedenberg, 2014) o que pode reduzir a biodisponibilidade do fármaco e contribuir para a variabilidade interindividual nas concentrações plasmáticas do fármaco (Nadaleto, Herbella, \& Patti, 2016). Logo, o ajuste de dose torna-se essencial para garantir a manutenção das concentrações plasmáticas dos antirretrovirais em níveis terapêuticos satisfatórios.

\section{Interações alimentares}

A interação entre fármaco e nutriente é a consequência da relação físico-química ou fisiológica entre um fármaco e uma macromolécula ou micronutriente, presentes em determinado alimento ou suplemento dietético (Won, Oberlies, \& Paine, 2012).

Essa interação pode comprometer a efetividade do tratamento em consequência de alterações na farmacocinética, influenciando negativamente parâmetros como a absorção, distribuição, metabolismo e excreção dos fármacos. Tais alterações 
podem aumentar o risco de reações adversas e toxicidade, ou, levar à falha terapêutica (Gauthier \& Malone, 1998; Lombardo \& Eserian, 2014).

Para que o objetivo terapêutico dos antirretrovirais seja atingido com maior sucesso e menor risco de toxicidade, é importante conhecer a melhor maneira de administração dos antirretrovirais. Por exemplo, é recomendada a administração de ritonavir, darunavir, etravirina e lamivudina com as refeições para melhorar a tolerabilidade gástrica e garantir a absorção oral máxima, evidenciando que o fármaco não possui alteração de absorção com os alimentos (D. S. de Souza, Hora, Padilha, Gonçalves, \& Peres, 2014). Do mesmo modo, o saquinavir e o nelfinavir apresentam um aumento da absorção em cerca de 2 a 3 vezes quando administrados com alimentos (Gauthier \& Malone, 1998; Nettles et al., 2006).

Antirretrovirais como amprenavir, lopinavir, tenofovir, zidovudina, efavirenz e ritonavir, também apresentam maior potencial de absorção quando administrados com alimentos gordurosos. É notável que nestas condições, o tenofovir e o lopinavir possuem suas concentrações plasmáticas elevadas em até 40\% 48\%, respectivamente (D. S. de Souza et al., 2014; Winston et al., 2005). Porém, o aumento da absorção do efavirenz gera também um aumento na frequência de reações adversas associadas ao sistema nervoso central. Desse modo é recomendada a administração com estômago vazio, de preferência antes de dormir (D. S. de Souza et al., 2014).

Outros antirretrovirais possuem problemas de absorção quando ingeridos concomitantes aos alimentos. O indinavir possui melhora na permeabilidade intestinal quando administrado em jejum (D. S. de Souza et al., 2014). A didanosina também possui recomendação de ser administrada 30 minutos antes ou 2 horas após as refeições para não ter sua absorção reduzida (D. S. de Souza et al., 2014). Embora a administração da didanosina com revestimento entérico seja recomendada sem alimento para melhorar a tolerância, algumas vezes, observa-se na prática clínica que este antirretroviral é prescrito com alimentos. López et al., (2006) defende que quando a adesão é superior a $80 \%$, a didanosina pode ser administrada com alimentos sem resultar em falha virológica.

\section{Uso e abuso de drogas lícitas e ilícitas}

Em 2015, aproximadamente 66,4\% da população brasileira entre 12 a 65 anos fizeram uso de bebida alcoólica, 33,5\% fizeram uso de cigarros industrializados e 17,3\% fizeram o uso de substâncias ilícitas alguma vez na vida (Bastos, Vasconcellos, De Boni, Reis, \& Coutinho, 2017).

Em pessoas vivendo com HIV, o uso de drogas psicoativas associado à administração de antirretrovirais pode resultar em interações de grande relevância clínica (Kumar, Rao, Earla, \& Kumar, 2015). A interação entre antirretrovirais e drogas psicoativas altera os transportadores de membrana e enzimas CYP450 do metabolismo hepático, as quais são responsáveis pelos mecanismos de eliminação de substâncias no organismo, reduzindo (inibição) ou potencializando (indução) as concentrações séricas dos medicamentos (Bracchi et al., 2015; Brasil, 2018; Khalsa, Talal, \& Morse, 2017; Santos, Secoli, \& Padoin, 2016; Stolbach, Paziana, Heverling, \& Pham, 2015).

O uso de álcool em associação com os antirretrovirais ocasiona na redução de células TCD4+ e aumento da carga viral devido ao estresse oxidativo gerado pelo CYP2E1, enzima responsável pela metabolização do álcool. Já a CYP3A4, enzima que metaboliza algumas classes de antirretrovirais, também é responsável pelo metabolismo da cocaína e seu metabólito (que é hepatotóxico). Desta forma, a associação desta droga com os antirretrovirais pode resultar em hepatotoxicidade grave, evolução da doença e piora do sistema imunológico. As drogas sintéticas como as anfetaminas e metanfetaminas são metabolizadas principalmente pelos CYP2D6 e CYP3A4 e podem interagir quando administradas com os antirretrovirais, resultando em toxicidade aguda (Bracchi et al., 2015; Brasil, 2018; Kumar et al., 2015). $\quad$ O abacavir pode ter seus níveis séricos elevado mais de 41\% se ingerido com álcool (Mcdowell, Chittick, Stevens, Edwards, \& Stein, 2000), já a 
didadosina se torna mais toxica aumentando a probabilidade do paciente desenvolver pancreatite (Oliveira, Ferreira, Yonamine, \& Chehter, 2014).

Apesar de poucos estudos sobre as interações causadas pelo uso da maconha, os medicamentos indinavir e nelfinavir podem apresentar concentrações reduzidas se usados concomitantemente. O uso de norvir com o ecstasy e/ou metanfetamina pode aumentar a concentração plasmática, enquanto, o uso com heroína reduz (Ferreira, 2004). Dessa forma, devido à complexidade das interações, torna-se importante realizar o monitoramento das concentrações plasmáticas para o ajuste de dose dos antirretrovirais em pacientes que fazem uso concomitante de drogas de abuso (Nuh, 2020).

\section{Polimorfismo genético}

Uma molécula de DNA é formada por milhares de genes, um gene é constituído por uma sequência específica de nucleotídeos. Essas sequências apresentam variações em diferentes indivíduos, quando a composição genética varia $1 \%$ ou mais é denominada polimorfismo genético (Evans \& Relling, 1999). O genoma humano possui aproximadamente 30 mil genes, totalizando 3,12 bilhões de nucleotídeos, os quais exibem mais de 12 milhões de polimorfismos genéticos (Venter et al., 2001). A variabilidade genética de enzimas ligadas ao metabolismo provoca alteração na ação do fármaco, fazendo-se necessário o ajuste de dose (Soeria-Atmadja et al., 2017).

Muitos polimorfismos genéticos ocorrem em enzimas do citocromo P450 (Calcagno, Cusato, D'Avolio, \& Bonora, 2017; Cressey \& Lallemant, 2007; Svärd, Spiers, Mulcahy, \& Hennessy, 2010), em transportadores como a glicoproteína P, em canais de cátions e íons (Eichelbaum, Ingelman-Sundberg, \& Evans, 2006; Klotz, Schwab, \& Treiber, 2004) e também em receptores nucleares como o receptor da vitamina D (Simões, Camera, Pacheco, \& Nascimento, 2020). Em pessoas vivendo com HIV que apresentaram mutação no gene CYP2B6 alelo G516T, verificou-se uma redução significativa na contagem de linfócitos TCD4+ com a administração de efavirenz. Nesse caso, recomenda-se o aumento da dose de efavirenz para que o fármaco seja efetivo (Queiroz et al., 2017). Em outro grupo envolvendo crianças vivendo com HIV que possuíam modificações nos genes CYP2B6 e CYP2A6, foi encontrado que 71,0\% não apresentavam a concentração plasmática de efavirenz dentro da faixa terapêutica recomenda, sendo necessário o aumento de dose do efavirenz (Soeria-Atmadja et al., 2017).

Outro estudo demonstrou que pacientes em uso de efavirenz com polimorfismos no gene da interleucina 10 (citocina que impede a replicação do vírus HIV na fase inicial da doença) apresentam maior predisposição à hepatotoxicidade associada aos antirretrovirais, sobretudo nos pacientes que adicionalmente fazem o uso de tabaco. No mesmo estudo, pacientes com o genótipo-819TT que já possuíam hepatotoxicidade exibiram maior risco de severidade da doença (Singh, Samani, Nain, \& Dhole, 2019). Neste aspecto, a farmacogenética ainda consiste em um campo de pesquisa incipiente, sendo necessária a realização de mais estudos visando preencher as lacunas existentes (Metzger, Souza-Costa, \& Tanus-Santos, 2006).

\section{Considerações Finais}

Diante da grande contribuição da terapia antirretroviral para a redução da morbimortalidade de pessoas vivendo com HIV, nota-se a relevância da realização de avaliações clínicas minuciosas no que se refere ao contexto fármaco-paciente. Esta revisão narrativa demonstra que grupos de indivíduos com condições fisiológicas específicas apresentam alterações em parâmetros farmacocinéticos como absorção, distribuição, metabolismo e excreção, assim como, variações dos níveis plasmáticos dos antirretrovirais e, portanto, destaca a importância do ajuste de dose para aumentar a efetividade dos antirretrovirais e proporcionar um tratamento mais adequado às pessoas vivendo com HIV. 
Para desenvolvimento futuro sugere-se estudos mais abrangentes em relação ao ajuste de doses de antirretrovirais em diferentes situações, visando sempre o melhor controle terapêutico. Aprofundando as análises de cada antirretroviral e o ajuste de dose necessário em situações especiais.

\section{Agradecimentos}

"O presente trabalho foi realizado com apoio da Coordenação de Aperfeiçoamento de Pessoal de Nível Superior Brasil (CAPES) - Código de Financiamento 001”.

\section{Referências}

AIDSINFO. (2018). Recommendations for the use of Antiretroviral Drugs in Pregnant Women with HIV Infection and Interventions to Reduce Perinatal HIV Transmission in the United States. https://aidsinfo.nih.gov/guidelines/htmltables/3/6656

AIDSINFO. (2019). Guidelines for the Use of Antiretroviral Agents in Pediatric HIV Infection. https://aidsinfo.nih.gov/guidelines/html/2/pediatricarv/50/maternal-hiv-testing-and-identification-of-perinatal-hiv-exposure

Araújo, P. D., \& Ehrhardt, A. (2015). Avaliação do dano hepático em pacientes HIV positivo sob terapia antirretroviral no município de Carazinho/RS. Saúde e Desenvolvimento Humano, 3(2), 45-54. https://doi.org/10.18316/2317-8582.15.4

Bastos, F. I. P. M., Vasconcellos, M. T. L. de, De Boni, R. B., Reis, N. B. dos, \& Coutinho, C. F. de S. (2017). III Levantamento Nacional sobre o uso de drogas pela população brasileira. ICICT/FIOCRUZ. ICICT/FIOCRUZ https://www.arca.fiocruz.br/handle/icict/34614

Benaboud, S., Hirt, D., Launay, O., Pannier, E., Firtion, G., Rey, E., \& Tréluyera, J. M. (2012). Pregnancy-related effects on tenofovir pharmacokinetics: A population study with 186 women. Antimicrobial Agents and Chemotherapy, 56(2), 857-862. https://doi.org/10.1128/AAC.05244-11

Benaboud, S., Tréluyer, J. M., Urien, S., Blanche, S., Bouazza, N., Chappuy, H., \& Hirt, D. (2012). Pregnancy-related effects on lamivudine pharmacokinetics in a population study with 228 women. Antimicrobial Agents and Chemotherapy, 56(2), 776-782. https://doi.org/10.1128/AAC.00370-11

Benedetti, M. S., Whomsley, R., \& Canning, M. (2007). Drug metabolism in the paediatric population and in the elderly. Drug Discovery Today, 12(15-16), 599-610. https://doi.org/10.1016/j.drudis.2007.06.011

Best, B. M., Mirochnick, M., Capparelli, E. V., Stek, A., Burchett, S. K., Holland, D. T., \& Connor, J. D. (2006). Impact of pregnancy on abacavir pharmacokinetics. Aids, Vol. 20, pp. 553-560. https://doi.org/10.1097/01.aids.0000210609.52836.d1

Bracchi, M., Stuart, D., Castles, R., Khoo, S., Back, D., \& Boffito, M. (2015). Increasing use of "party drugs" in people living with HIV on antiretrovirals: A concern for patient safety. AIDS, 29(13), 1585-1592. https://doi.org/10.1097/QAD.0000000000000786

Brasil. Lei $n^{\circ}$ 9313, de 13 de novembro de 1996. Dispões sobre a distribuição gratuita de medicamentos aos portadores do HIV e doentes de AIDS. , (1996). Brasília - DF: Diario Oficial da União.

Brasil. (2010). Adesão ao Tratamento Antirretroviral no Brasil: Coletânea de Estudos do Projeto Atar. Retrieved Ministério da Saúde, Secretaria de Vigilância em Saúde Departamento de DST, Aids e Hepatites Virais. https://www.nescon.medicina.ufmg.br/biblioteca/imagem/3035.pdf

Brasil. (2018). Protocolo Clínico e Diretrizes Terapêuticas para Manejo da Infecção pelo HIV em Adultos. http://www.aids.gov.br/pt-br/pub/2013/protocoloclinico-e-diretrizes-terapeuticas-para-manejo-da-infeccao-pelo-hiv-em-adultos

BRASIL. (2019). Boletim epidemiológico. Brasília. http://www.aids.gov.br/pt-br/pub/2019/boletim-epidemiologico-de-hivaids-2019

Brito, D. M. S. de. (2012). Guia de cuidados aos pacientes em uso de terapia antirretroviral.

Calcagno, A., Cusato, J., D’Avolio, A., \& Bonora, S. (2017). Genetic Polymorphisms Affecting the Pharmacokinetics of Antiretroviral Drugs. Clinical Pharmacokinetics, 56(4), 355-369. https://doi.org/10.1007/s40262-016-0456-6

Canals, F., Masiá, M., \& Gutiérrez, F. (2018). Developments in early diagnosis and therapy of HIV infection in newborns. Expert Opinion on Pharmacotherapy, 19(1), 13-25. https://doi.org/10.1080/14656566.2017.1363180

Capparelli, E. V., Mirochnick, M., Dankner, W. M., Blanchard, S., Mofenson, L., McSherry, G. D., \& Oram, R. (2003). Pharmacokinetics and tolerance of zidovudine in preterm infants. Journal of Pediatrics, 142(1), 47-52. https://doi.org/10.1067/mpd.2003.mpd0335

CDC. (2017, August). HIV infection and older Americans: The public health perspective. American Journal of Public Health, 102, 1516-1526. https://doi.org/10.2105/AJPH.2012.300844

Chang, P., \& Friedenberg, F. (2014). Obesity and GERD. Gastroenterology Clinics of North America, 43(1), 161-173. https://doi.org/10.1016/j.gtc.2013.11.009

Chastain, D. B., Henderson, H., \& Stover, K. R. (2015). Epidemiology and Management of Antiretroviral-Associated Cardiovascular Disease. The Open AIDS Journal, 9(1), 23-37. https://doi.org/10.2174/1874613601509010023

Connor, E. M., Sperling, R. S., Gelber, R., Kiselev, P., Scott, G., O’sullivan, M. jo, \& Balsley, J. (1994). Reduction of maternal-infant transmission of human 
immunodeficiency virus type 1 with zidovudine treatment. New England Journal of Medicine, 331(18), 1173-1180. https://doi.org/10.1056/NEJM199411033311801

Costantine, M. M. (2014). Physiologic and pharmacokinetic changes in pregnancy. Frontiers in Pharmacology, 5 APR(April), 1-5. https://doi.org/10.3389/fphar.2014.00065

Courlet, P., Stader, F., Guidi, M., Saldanha, S. A., Stoeckle, M., Cavassini, M., \& Marzolini, C. (2019). Pharmacokinetic profiles of boosted darunavir, dolutegravir and lamivudine in aging patients enrolled in the Swiss HIV Cohort Study. Aids, 1. https://doi.org/10.1097/qad.0000000000002372

Cressey, T. R., \& Lallemant, M. (2007). Pharmacogenetics of antiretroviral drugs for the treatment of HIV-infected patients: An update. Infection, Genetics and Evolution, Vol. 7, pp. 333-342. Elsevier. https://doi.org/10.1016/j.meegid.2006.08.004

Dawes Bsc, M., \& Chowienczyk Bsc, P. J. (2001). Pharmacokinetics in pregnancy Lecturer in Clinical Pharmacology. 15(6), 819-826. https://doi.org/10.1053/beog.2001.0231

De Sousa Mendes, M., \& Chetty, M. (2019). Are Standard Doses of Renally-Excreted Antiretrovirals in Older Patients Appropriate: A PBPK Study Comparing Exposures in the Elderly Population With Those in Renal Impairment. Drugs in R and D, (0123456789). https://doi.org/10.1007/s40268-01900285-0

Dupin, N., Buffet, M., Marcelin, A. G., Lamotte, C., Gorin, I., Ait-Arkoub, Z., \& Peytavin, G. (2002). HIV and antiretroviral drug distribution in plasma and fat tissue of HIV-infected patients with lipodystrophy. AIDS, 16(18), 2419-2424. https://doi.org/10.1097/00002030-200212060-00006

Eichelbaum, M., Ingelman-Sundberg, M., \& Evans, W. E. (2006). Pharmacogenomics and individualized drug therapy. Annual Review of Medicine, 57, 119137. https://doi.org/10.1146/annurev.med.56.082103.104724

Ekrikpo, U. E., Kengne, A. P., Bello, A. K., Effa, E. E., Noubiap, J. J., Salako, B. L., \& Okpechi, I. G. (2018). Chronic kidney disease in the global adult HIVinfected population: A systematic review and meta-analysis. PLOS ONE, 13(4), 1-24. https://doi.org/10.1371/journal.pone.0195443

Ellis, C. L. (2017). HIV associated kidney diseases: Clarifying concordance between renal failure in HIV infection and histopathologic manifestations at kidney biopsy. Seminars in Diagnostic Pathology, 34(4), 377-383. https://doi.org/10.1053/j.semdp.2017.04.009

Estrela, C. (2018). Metodologia Científica: Ciência, Ensino, Pesquisa. Editora Artes Médicas.

Evans, W. E., \& Relling, M. V. (1999, October 15). Pharmacogenomics: Translating functional genomics into rational therapeutics. Science, 286(5439), 487491. https://doi.org/10.1126/science.286.5439.487

Ferreira, V. M. B. (2004). Álcool, Drogas Ilícitas E Anti-Retrovirais. https://www.saude.sc.gov.br/index.php/informacoes-gerais-documentos/conferencias-eencontros/conferencia-estadual-de-saude-mental/textos/3163-alcool-droga-ilicita/file

Friedl, D. B., Aprile, D. C. B., Fino, L. C., Barbosa, D. A., \& Belasco, A. G. S. (2009). Evolução da função renal de pacientes portadores do Vírus da Imunodeficiência Humana/ Síndrome da Imunodeficiência Adquirida. Acta Paulista de Enfermagem, 22(spe1), 531-534. https://doi.org/10.1590/s010321002009000800016

Ganesan, M., Poluektova, L. Y., Kharbanda, K. K., \& Osna, N. A. (2018). Liver as a target of human immunodeficiency virus infection. World Journal of Gastroenterology, 24(42), 4728-4737. https://doi.org/10.3748/wjg.v24.i42.4728

Gauthier, I., \& Malone, M. (1998). Drug-food interactions in hospitalised patients. Drug Safety, 18(6), 383-393. https://doi.org/10.2165/00002018199818060-00001

Gilbert, E. M., Darin, K. M., Scarsi, K. K., \& McLaughlin, M. M. (2015). Antiretroviral pharmacokinetics in pregnant women. Pharmacotherapy, 35(9), 838855. https://doi.org/10.1002/phar.1626

Guaraldi, G., Pintassilgo, I., Milic, J., \& Mussini, C. (2018). Managing antiretroviral therapy in the elderly HIV patient. Expert Review of Clinical Pharmacology, 11(12), 1171-1181. https://doi.org/10.1080/17512433.2018.1549484

Hall, A. M., Hendry, B. M., Nitsch, D., \& Connolly, J. O. (2011). Tenofovir-associated kidney toxicity in HIV-infected patients: A review of the evidence. American Journal of Kidney Diseases, 57(5), 773-780. https://doi.org/10.1053/j.ajkd.2011.01.022

Hürlimann, D., Chenevard, R., Ruschitzka, F., Flepp, M., Enseleit, F., Béchir, M., \& Weber, R. (2006). Effects of statins on endothelial function and lipid profile in HIV infected persons receiving protease inhibitor-containing anti-retroviral combination therapy: A randomised double blind crossover trial. Heart, 92(1), 110-112. https://doi.org/10.1136/hrt.2004.056523

Ishizaki, A., Cuong, N. H., Thuc, P. Van, Trung, N. V., Saijoh, K., Kageyama, S., \& Ichimura, H. (2009). Profile of HIV type 1 infection and genotypic resistance mutations to antiretroviral drugs in treatment-naive HIV type 1-infected individuals in Hai Phong, Viet Nam. AIDS Research and Human Retroviruses, 25(2), 175-182. https://doi.org/10.1089/aid.2008.0193

Jain, R., Chung, S. M., Jain, L., Khurana, M., Lau, S. W. J., Lee, J. E., \& Sahajwalla, C. G. (2011). Implications of obesity for drug therapy: Limitations and challenges. Clinical Pharmacology and Therapeutics, 90(1), 77-89. https://doi.org/10.1038/clpt.2011.104

Jourjy, J., Dahl, K., \& Huesgen, E. (2015). Antiretroviral treatment efficacy and safety in older HIV-infected adults. Pharmacotherapy, 35(12), 1140-1151. https://doi.org/10.1002/phar.1670

Kaspar, M. B., \& Sterling, R. K. (2017). Mechanisms of liver disease in patients infected with HIV. BMJ Open Gastroenterology, 4(1), 1-7. https://doi.org/10.1136/bmjgast-2017-000166

Khalsa, J. H., Talal, A. H., \& Morse, G. (2017). Drug-Drug Interactions and Diagnostics for Drug Users With HIV and HIV/HCV Coinfections: Introduction. Clinical Pharmacology in Drug Development, 6(2), 108-117. https://doi.org/10.1002/cpdd.316 
Kim, M. H., Ahmed, S., \& Abrams, E. J. (2016). Paediatric HIV: Progress on Prevention, Treatment and Cure. 3(3), 219-229. https://doi.org/10.1007/s40124-015-0087-7.Paediatric

King, J. R., Kimberlin, D. W., Aldrovandi, G. M., \& Acosta, E. P. (2002). Antiretroviral Pharmacokinetics in the Paediatric Population A Review. Infancy, $41(14), 1115-1133$

Klotz, U., Schwab, M., \& Treiber, G. (2004). CYP2C19 polymorphism and proton pump inhibitors. Basic and Clinical Pharmacology and Toxicology, 95(1), 2-8. https://doi.org/10.1111/j.1600-0773.2004.pto950102.x

Ku, C. L., \& Smith, B. P. (2011). Dosing in neonates: Special considerations in physiology and trial design. Bone, 23(1), 1-7. https://doi.org/10.1038/jid.2014.371

Kumar, S., Rao, P. S. S., Earla, R., \& Kumar, A. (2015). Drug-drug interactions between anti-retroviral therapies and drugs of abuse in HIV systems. Expert Opinion on Drug Metabolism and Toxicology, 11(3), 343-355. https://doi.org/10.1517/17425255.2015.996546

Lombardo, M., \& Eserian, J. K. (2014). Fármacos E Alimentos: Interações E Influências Na Terapêutica. Infarma - Ciências Farmacêuticas, 26(3), 188-192. https://doi.org/10.14450/2318-9312.v26.e3.a2014.pp188-192

López, J. C., Moreno, S., Jiménez-Oñate, F., Clotet, B., Rubio, R., \& Hernández-Quero, J. (2006). A cohort study of the food effect on virological failure and treatment discontinuation in patients on HAART containing didanosine enteric-coated capsules (FOODDle study). HIV Clinical Trials, 7(4), 155-162. https://doi.org/10.1310/hct0704-155

Madelain, V., Le, M. P., Champenois, K., Charpentier, C., Landman, R., Joly, V., \& Peytavin, G. (2017). Impact of obesity on antiretroviral pharmacokinetics and immunovirological response in HIV-infected patients: A case-control study. Journal of Antimicrobial Chemotherapy, 72(4), 1137-1146. https://doi.org/10.1093/jac/dkw527

Mangoni, A. A., \& Jackson, S. H. D. (2004). Age-related changes in pharmacokinetics and pharmacodynamics: Basic principles and practical applications. British Journal of Clinical Pharmacology, 57(1), 6-14. https://doi.org/10.1046/j.1365-2125.2003.02007.x

Mcdowell, J. A., Chittick, G. E., Stevens, C. P., Edwards, K. D., \& Stein, D. S. (2000). Pharmacokinetic interaction of abacavir (1592U89) and ethanol in human immunodeficiency virus-infected adults. Antimicrobial Agents and Chemotherapy, 44(6), 1686-1690. https://doi.org/10.1128/AAC.44.6.16861690.2000

Medeiros, J. L., \& Ferreira, M. L. H. (1998). Cirrose Hepática e Insuficiência hepática. Terapêutica Clínica. Guanabara Koogan.

Menezes, A. M., Torelly, J., Real, L., Bay, M., Poeta, J., \& Sprinz, E. (2011). Prevalence and risk factors associated to chronic kidney disease in HIV-infected patients on HAART and undetectable viral load in Brazil. PLOS ONE, 6(10). https://doi.org/10.1371/journal.pone.0026042

Metzger, I. F., Souza-Costa, D. C., \& Tanus-Santos, J. E. (2006). Farmacogenética: Princípios, aplicações e perspectivas. Medicina, 39(4), 515-521. https://doi.org/10.11606/issn.2176-7262.v39i4p515-521

Mocroft, A., Kirk, O., Reiss, P., De Wit, S., Sedlacek, D., Beniowski, M., \& EuroSIDA Study Group. (2010). Estimated glomerular filtration rate, chronic kidney disease and antiretroviral drug use in HIV-positive patients. AIDS, 24(11), 1667-1678. https://doi.org/10.1097/QAD.0b013e328339fe53

Nadaleto, B. F., Herbella, F. A. M., \& Patti, M. G. (2016). Gastroesophageal reflux disease in the obese: Pathophysiology and treatment. Surgery (United States), 159(2), 475-486. https://doi.org/10.1016/j.surg.2015.04.034

Naicker, S., Rahmanian, S., \& Kopp, J. B. (2015). HIV and chronic kidney disease. Clinical Nephrology, 83, S32-S38. https://doi.org/10.5414/CNP83S032

NCBI. (2019). National Center for Biotechnology Information. https://www.ncbi.nlm.nih.gov/

Neto, L. F. da S. P., Bassetti, B. R., Fraga, I. H. V., Oliveira Santos, C. R., Ximenes, P. D., \& Miranda, A. E. (2016). Nephrotoxicity during tenofovir treatment: A three-year follow-up study in a Brazilian reference clinic. Brazilian Journal of Infectious Diseases, 20(1), 14-18. https://doi.org/10.1016/j.bjid.2015.09.004

Nettles, R. E., Kieffer, T. L., Parsons, T., Johnson, J., Cofrancesco, J., Gallant, J. E., \& Flexner, C. (2006). Marked intraindividual variability in antiretroviral concentrations may limit the utility of therapeutic drug monitoring. Clinical Infectious Diseases, 42(8), 1189-1196. https://doi.org/10.1086/501458

Nuh, O. (2020). Interactions between HIV treatment and recreational drugs. https://www.aidsmap.com/about-hiv/interactions-between-hiv-treatment-andrecreational-drugs

Nunes, E. P. (2016). Terapia antirretroviral e função renal. The Brazilian Journal of Infectious Diseases, 2(3), 82-90. https://www.bjid.org.br/en-terapiaantirretroviral-e-funcao-renal-articulo-X217751171655969X

Núñez, M. (2010). Clinical syndromes and consequences of antiretroviral-related hepatotoxicity. Hepatology, 52(3), 1143-1155. https://doi.org/10.1002/hep.23716

Oliveira, N. M., Ferreira, F. A. Y., Yonamine, R. Y., \& Chehter, E. Z. (2014). Drogas antirretrovirais e pancreatite aguda em pacientes com HIV/AIDS: existe alguma associação? Revisão da literatura. Einstein, 12(1). https://www.scielo.br/j/eins/a/kdTPqNZS7Ymkn7MfW5kX6Sv/?lang=pt\&format=pdf

Pacifici, G. M. (2005). Pharmacokinetics of antivirals in neonate. Early Human Development, 81(9), 773-780. https://doi.org/10.1016/j.earlhumdev.2005.06.001

Pariente, G., Leibson, T., Carls, A., Adams-Webber, T., Ito, S., \& Koren, G. (2016). Pregnancy-Associated Changes in Pharmacokinetics: A Systematic Review. PLoS Medicine, 13(11), 1-36. https://doi.org/10.1371/journal.pmed.1002160 
Pezzani, M. D., Resnati, C., Di Cristo, V., Riva, A., \& Gervasoni, C. (2016). Abacavir-induced liver toxicity. Brazilian Journal of Infectious Diseases, 20(5), 502-504. https://doi.org/10.1016/j.bjid.2016.03.002

Post, F. A., \& Holt, S. G. (2009). Recent developments in HIV and the kidney. Current Opinion in Infectious Diseases, 22(1), 43-48. https://doi.org/10.1097/QCO.0b013e328320ffec

Queiroz, M. A. F., Laurentino, R. V., Amoras, E. da S. G., Araújo, M. S. M. de, Gomes, S. T. M., Lima, S. S., \& Machado, L. F. A. (2017). The CYP2B6 G516T polymorphism influences CD4+ T-cell counts in HIV-positive patients receiving antiretroviral therapy in an ethnically diverse region of the Amazon. International Journal of Infectious Diseases, 55, 4-10. https://doi.org/10.1016/j.ijid.2016.12.002

Rakhmanina, N., \& Phelps, B. R. (2012). Pharmacotherapy of Pediatric HIV Infection. Pediatric Clinics of North America, 59(5), 1093-1115. https://doi.org/10.1016/j.pcl.2012.07.009

Raposeiras-Roubín, S., \& Triant, V. (2016). Ischemic Heart Disease in HIV: An In-depth Look at Cardiovascular Risk. Revista Española de Cardiología (English Edition), 69(12), 1204-1213. https://doi.org/10.1016/j.rec.2016.10.005

Reynes, J., Pevriere, H., De Boever, C., \& Le Moing, V. (2003). Renal tubular injury and severe hypophosphoremia (Fanconi syndrome) associated with tenofovir therapy [poster 717]. In Program an Abstracts of the 10th Conference on Retroviruses and Opportunistic Infections. Boston, USA. Alexandria, VA: Foundation for Retrovirology and Human Health.

Ritschel, W. A., \& Kearns, G. L. (2009). Handbook of Basic Pharmacokinetics Including Clinical Applications (7a ed.). The American Pharmacists Association. https://doi.org/10.21019/9781582121260

Rodighiero, V. (1989). Effects Of Cardiovascular Disease On Pharmacokinetics Summary. Cardiovascular Drugs and Therapy, 3, 711-730.

Rongkavilit, C., Thaithumyanon, P., Chuenyam, T., Damle, B. D., Limpongsanurak, S., Boonrod, C., \& Phanuphak, P. (2001). Pharmacokinetics of stavudine and didanosine coadministered with nelfinavir in human immunodeficiency virus-exposed neonates. Antimicrobial Agents and Chemotherapy, 45(12), 35853590. https://doi.org/10.1128/AAC.45.12.3585-3590.2001

Santos, W. M. Dos, Secoli, S. R., \& Padoin, S. M. de M. (2016). Potential drug interactions in patients given antiretroviral therapy. Revista Latino-Americana de Enfermagem, 24. https://doi.org/10.1590/1518-8345.1193.2832

Schijvens, A. M., Wildt, S. N. de, \& Schreuder, M. F. (2020). Pharmacokinetics in children with chronic kidney disease. Pediatric Nephrology, 35(7), 11531172. https://doi.org/10.1007/s00467-019-04304-9

Schoen, J. C., Erlandson, K. M., \& Anderson, P. L. (2013). Clinical pharmacokinetics of antiretroviral drugs in older persons. Expert Opinion on Drug Metabolism and Toxicology, 9(5), 573-588. https://doi.org/10.1517/17425255.2013.781153

Simões, G. L. D. B. A., Camera, P., Pacheco, A., \& Nascimento, R. E. do. (2020). Polimorfismos genéticos no receptor da vitamina D e a suscetibilidade a doenças no Brasil. Revista de Patologia Do Tocantins, 7(2), 83-87. https://doi.org/10.20873/uft.2446-6492.2020v7n2p83

Singh, H. O., Samani, D., Nain, S., \& Dhole, T. N. (2019). Interleukin-10 polymorphisms and susceptibility to ARV associated hepatotoxicity. Microbial Pathogenesis, 133. https://doi.org/10.1016/j.micpath.2019.103544

Smit, C., Hoogd, S. de, Brüggemann, R. J. M., \& Knibbe, C. A. J. (2018). Obesity and drug pharmacology: a review of the influence of obesity on pharmacokinetic and pharmacodynamic parameters. Expert Opinion on Drug Metabolism and Toxicology, 14(3), 275-285. https://doi.org/10.1080/17425255.2018.1440287

Soeria-Atmadja, S., Österberg, E., Gustafsson, L. L., Dahl, M. L., Eriksen, J., Rubin, J., \& Navér, L. (2017). Genetic variants in CYP2B6 and CYP2A6 explain interindividual variation in efavirenz plasma concentrations of HIV-infected children with diverse ethnic origin. PLoS ONE, 12(9). https://doi.org/10.1371/journal.pone.0181316

Souza, D. S. de, Hora, T. F. da, Padilha, P. de C., Gonçalves, J. C. S., \& Peres, W. A. F. (2014). Efeito dos alimentos na biodisponibilidade de medicamentos antirretrovirais: uma revisão sistemática da literatura. Nutrire, 39(2), 243-251. https://doi.org/10.4322/nutrire.2014.023

Souza, J. de, \& Storpirtis, S. (2004). Antiretroviral activity and pharmacokinetics properties of lamivudine and zidovudine association. Revista Brasileira de Ciencias Farmaceuticas/Brazilian Journal of Pharmaceutical Sciences, 40(1), 9-19. https://doi.org/10.1590/s1516-93322004000100004

Spina, E., Santoro, V., \& D’Arrigo, C. (2008). Clinically relevant pharmacokinetic drug interactions with second-generation antidepressants: An update. Clinical Therapeutics, 30(7), 1206-1227. https://doi.org/10.1016/S0149-2918(08)80047-1

Stader, F., Kinvig, H., Penny, M. A., Battegay, M., Siccardi, M., \& Marzolini, C. (2019). Physiologically Based Pharmacokinetic Modelling to Identify Pharmacokinetic Parameters Driving Drug Exposure Changes in the Elderly. Clinical Pharmacokinetics, (0123456789). https://doi.org/10.1007/s40262-01900822-9

Stolbach, A., Paziana, K., Heverling, H., \& Pham, P. (2015). A Review of the Toxicity of HIV Medications II: Interactions with Drugs and Complementary and Alternative Medicine Products. Journal of Medical Toxicology, 11(3), 326-341. https://doi.org/10.1007/s13181-015-0465-0

Sudano, I., Spieker, L. E., Noll, G., Corti, R., Weber, R., \& Lüscher, T. F. (2006). Cardiovascular disease in HIV infection. American Heart Journal, 151(6), 1147-1155. https://doi.org/10.1016/j.ahj.2005.07.030

Sulkowski, M. S., Thomas, D. L., Mehta, S. H., Chaisson, R. E., \& Moore, R. D. (2002). Hepatotoxicity associated with nevirapine or efavirenz-containing antiretroviral therapy: Role of hepatitis C and B infections. Hepatology, 35(1), 182-189. https://doi.org/10.1053/jhep.2002.30319

Svärd, J., Spiers, J. P., Mulcahy, F., \& Hennessy, M. (2010). Nuclear receptor-mediated induction of CYP450 by antiretrovirals: Functional consequences of NR1I2 (PXR) polymorphisms and differential prevalence in whites and sub-Saharan Africans. Journal of Acquired Immune Deficiency Syndromes, 55(5), 536-549. https://doi.org/10.1097/QAI.0b013e3181f52f0c 
Swanepoel, C. R., Atta, M. G., D’Agati, V. D., Estrella, M. M., Fogo, A. B., Naicker, S., \& Zeier, M. (2018). Kidney disease in the setting of HIV infection: conclusions from a Kidney Disease: Improving Global Outcomes (KDIGO) Controversies Conference. Kidney International, 93(3), 545-559. https://doi.org/10.1016/j.kint.2017.11.007

Terada, T., \& Hira, D. (2015). Intestinal and hepatic drug transporters: pharmacokinetic, pathophysiological, and pharmacogenetic roles. Journal of Gastroenterology, 50(5), 508-519. https://doi.org/10.1007/s00535-015-1061-4

Thapa, R. K., Choi, H. G., Kim, J. O., \& Yong, C. S. (2017). Analysis and optimization of drug solubility to improve pharmacokinetics. Journal of Pharmaceutical Investigation, 47(2), 95-110. https://doi.org/10.1007/s40005-016-0299-z

Triant, V. A. (2013). Cardiovascular Disease and HIV Infection Virginia. Curr HIV/AIDS, 10(3), 199-206. https://doi.org/10.1007/s11904-013-01686.Cardiovascular

UNAIDS. (2019a). Joint United Nations Programme on HIV/AIDS. https://www.unaids.org/en/regionscountries/countries/brazil

UNAIDS. (2019b). Joint United Nations Programme on HIV/AIDS. https://www.unaids.org/en/resources/documents/2019/2019-UNAIDS-d

UNAIDS. (2020). Joint United Nations Programme on HIV/AIDS. https://www.unaids.org/en/resources/documents/2020

Venter, J. C., Adams, M. D., Myers, E. W., Li, P. W., Mural, R. J., Sutton, G. G., \& Zhu, X. (2001). The sequence of the human genome. Science, 291(5507), 1304-1351. https://doi.org/10.1126/science. 1058040

Verma, S., \& Kaplowitz, N. (2009). Diagnosis, management and prevention of drug-induced liver injury. General Medicine, 58(11), 1555-1564. https://doi.org/10.1136/gut.2008.163675

Violari, A., PH, D., Steyn, J., Sc, M., Madhi, S. A., Paed, F. C., \& Jean-philippe, P. (2008). Violari A, Cotton MF, Gibb DM, et al. Early antiretroviral therapy and mortality among HIV-infected infants. N Engl J Med. Nov 2008;359(21):2233-2244. 359(21), 2233-2244. https://doi.org/10.1056/NEJMoa0800971.Early

Winston, A., Bloch, M., Carr, A., Amin, J., Mallon, P. W. G., Ray, J., \& Emery, S. (2005). Atazanavir trough plasma concentration monitoring in a cohort of HIV-1-positive individuals receiving highly active antiretroviral therapy. Journal of Antimicrobial Chemotherapy, 56(2), 380-387. https://doi.org/10.1093/jac/dki235

Winter, M. E. (2004). Basic clinical pharmacokinetics (4th ed). Lippincott Williams \& Wilkins, c2004.

Won, C. S., Oberlies, N. H., \& Paine, M. F. (2012). Mechanisms underlying food-drug interactions: Inhibition of intestinal metabolism and transport. Pharmacology and Therapeutics, 136(2), 186-201. https://doi.org/10.1016/j.pharmthera.2012.08.001

Yellepeddi, V. (2016). Princípios da terapia medicamentosa (6a ed.). Whalen, K. et al. Farmacologia Ilustrada. 